Journal of Applied Fluid Mechanics, Vol. 9, Special Issue 1, pp. 11-17, 2016.

Selected papers from the $7^{\text {th }}$ International Exergy, Energy

and Environment Symposium, IEEE7-2015

Available online at www.jafmonline.net, ISSN 1735-3572, EISSN 1735-3645.

DOI: $10.36884 /$ jafm.9.SI1.25825

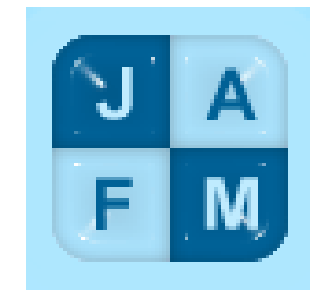

\title{
Turbulent Plane Impinging Jet-Physical Insight and Turbulence Modeling
}

\author{
M. Charmiyan ${ }^{1}{ }^{\dagger}$, A. R. Azimian ${ }^{2}$, L. KeirsbulcK ${ }^{3}$, E. Shirani ${ }^{4}$, F. Aloui ${ }^{3}$ \\ ${ }^{1}$ Isfahan University of Technology, Ph.D Candidate, Department of Mechanical Engineering, Isfahan, \\ 8415683111, Iran \\ ${ }^{2}$ Isfahan University of Technology, Professor, Department of Mechanical Engineering, Isfahan, \\ 8415683111, Iran \\ ${ }^{3}$ University of Lille North of France, UVHC, Professor, Campus Mont Houy, LAMIH, CNRS UMR 8201, \\ F-59313, Valenciennes Cedex 9, France \\ ${ }^{4}$ Foolad Institute of Technology, Professor, Department of Mechanical Engineering, Fooladshahr, Isfahan, \\ 84916 63763, Iran
}

†Corresponding Author Email:m.charmiyan@gmail.com

(Received October 20, 2015; accepted December 10, 2015)

\begin{abstract}
A 3D numerical simulation using large eddy simulation (LES) method is performed for a submerged turbulent water slot jet impinging normally on a flat plate with a nozzle-to-plate distance of 10 jet width and a Reynolds number of 16000 and the results are compared with the existing experimental data. The numerical platform is an open source CFD code based on the field operation and manipulation C ++ class library for continuum mechanics (OpenFOAM) and is used to simulate the flow and represent the mean and instantaneous flow field characteristics. Also, simulations are performed with two different subgrid-scale (SGS) models, one-equation based subgrid-scale model and localized dynamic smagorinsky model. Evaluating the different subgrid-scale (SGS) models, a priori and a posteriori test is done. Comparison between results obtained using the SGS models and experimental data shows that the simulation results using localized dynamic Smagorinsky model are more compatible with the experimental data compared with those that obtained from the kinetic energy one-equation model especially in regions close to the impingement wall and in free jet region.
\end{abstract}

Keywords: Impinging jet; Large eddy simulation; Turbulence modeling; Hybrid LES/RANS; Turbulent plane jet.

\section{Nomenclature}

C

Smagorinsky parameter

CFL Courant-Friedrich-Lewis

e slot nozzle width

gravitational constant

$H \quad$ nozzle to plate distance

$k \quad$ turbulent kinetic energy

$L_{i j} \quad$ Leonard stress tensor

$L_{x}, L_{y}, L_{z} \quad$ computational domain size

$p \quad$ instantaneous pressure

Re Reynolds number

$S_{i j} \quad$ strain rate

$t \quad$ time

$u_{i} \quad$ instantaneous velocity vector

$u_{i}^{\prime} \quad$ unresolved velocity vector

$U_{m} \quad$ average velocity at the exit of the nozzle

$\begin{array}{ll}\begin{array}{ll}\text { Greek letters } \\ \Delta r^{+},\end{array} & \text {non-dimensional size of the grid in } \\ (r \Delta \theta)^{+} \Delta z^{+} & \text {three cylindrical polar directions } \\ \mu & \text { dynamic viscosity } \\ \tau_{i j} & \text { subgrid stress } \\ \tau_{w} & \text { wall friction } \\ \Omega & \text { computational domain } \\ \text { Superscripts } & \\ +- & \text { dimensionless } \\ - & \text { first filter } \\ - & \text { second filter } \\ \text { Subscripts } & \\ \text { eff } & \text { effective } \\ I & \text { index of coordinate direction } \\ \text { max } & \text { maximum } \\ \text { turb } & \text { turbulence }\end{array}$




\section{Introduction}

Fluid flow and heat transfer in impinging jets have been widely studied because of their fundamental, as well as practical, relevance. Jet impingement has the maximum heat transfer rate among all the single-phase heat transfer processes, which leads to its wide industrial use in heating, cooling, and drying industries. (Dewan et al. 2012)

The physical aspects of such flows are highly complex and are still not fully understood especially at high Reynolds numbers, where experimental techniques are limited. Large-scale structures from the jet region of the flow can have a significant effect on the near-wall flow and in many cases drive the wall shear layer. Hence one of the key features to predict such flows is the interaction of the outer vortices with the wall shear layer. Due to better understanding of transfer mechanisms in turbulent impinging jet flows, a vast number of numerical and experimental investigations were done by a number of researchers. They found that the flow characteristics and wall heat transfer of turbulent impinging jets depend strongly on a number of aspects, such as confinement, nozzle-to-plate spacing (H/D), nozzle geometry and flow conditions at the nozzle outlet (in-flow conditions).

Various studies dealing with experimental and computational studies on fluid flow and heat transfer behaviour of impinging jets have been reported in the literature. Some of the important parameters that have been studied include nozzle to plate spacing, inflow turbulence, nozzle cross-section (circular or slot) and Reynolds number. Gardon and Akfirat (1965) studied the effects of inflow turbulence and nozzle to plate spacing on the heat transfer at the wall in a jet impinging flow. Jambunathan et al. (1992) reviewed the existing literature on experimental studies on Nusselt number predictions by an impinging circular jet. Martin (1977) also reviewed the experimental studies on single and multiple jet impinging flows.

The use of an efficient turbulent method is totally depends on the flow regime. Depending on the distance between the nozzle and impingement plate, there may exist various flow regimes for impingement flows. For small nozzle to plate distance $(\mathrm{H} / \mathrm{e})$, where the flow in the impact zone is physically laminar, the prediction of the shear stress and heat transfer levels in the stagnation flow region basically agree with the Reynolds-averaged Navier-Stokes, RANS, models due to use of stress limiters which damp most of the turbulence in the impact zone (Kubacki et al., 2013). the Reynolds Average Navier-Stokes (RANS) models have shown their limits to predict the impinging jet flow: (i) erroneous prediction of the stagnation zone flow, (ii) over prediction of the location of stagnation point heat transfer, (iii) large number of arbitrary coefficients leading to poor reproducibility of the models (iv) the transition from laminar to turbulent state in the developing boundary layer on the plate is completely ignored by RANS models. (Zuckerman and Lior, 2006).

In the last decade, unsteady numerical simulations (LES/DNS) of impinging jet flow have been carried out. Hallqvist (2006) investigated the flow structure using LES method. He did not use any explicit SGS model and instead handled it by using a fine grid resolution and upwind biased numerical scheme. Saving in computational resources, obtained by not considering any explicit SGS model, enabled him to construct a grid having twice as many cells. He argued that the application of an SGS model, which is typically incapable of handling anisotropy and backscatter, is more harmful than the error introduced by neglecting the SGS terms.

Olsson and Fuchs (1998) and Lodato et al. (2009) studied the possible effects of different SGS models on the accuracy of an impinging jet computation. They found that the prediction by different SGS models differed only in turbulence statistics and not in the mean velocity. Lodato et al. (2009) found that their new model (a mixed similarity with a WALE) performs quite well near the wall, especially in the stagnation region while, other two models (standard WALE and the Lagrangian dynamic Smagorinsky model) overestimated the streamwise turbulent fluctuations in this region. They attributed the accuracy of this new model to accurate representation of $t$ he backscatter of energy.

One of the most important factors that have the significant effect of the computation results is computational grid resolution. Grid sensitivity of numerical results has been investigated by a numbers of researchers. Piomelli and Chasnov (1996) have given prior meshing criteria for wall attaching flows, which requires $\Delta \mathrm{r}+<100$, $(\mathrm{r} \Delta \theta)+<20$, and $\Delta \mathrm{z}^{+<2}$. But, as reported by Hadziabdic and Hanjalic (2008), this requirement is not sufficient for impinging flows, especially in the radial direction. This is due to the strong acceleration and deceleration of the flow near the stagnation point. Lodato et al. (2009) also discussed grid requirements in LES of impinging flows.

As already mentioned, nozzle outlet conditions affect flow field and heat transfer in impinging flows and therefore their inaccurate specification can lead to inaccurate predictions of flow and heat transfer characteristics.

Among different LES investigations of impinging flows, Cziesla et al. (2001) used a uniform inlet velocity profile. Beaubert and Viazzo (2003) and Yu et al. (2005) obtained time-invariant inflow velocity profiles from the corresponding experiments. Lodato et al. (2009) used a turbulent power-law profile and superimposed it with a random noise. Voke and Gau (1998) and Hadziabdic and Hanjalic (2008) generated inflow conditions from precursor simulations of a channel flow and pipe flow, respectively, for their plane and round jet impingements. Rhea et al. (2009) and Uddin (2008) used a digital filter-based inflow turbulence 
generator to add fluctuations to the mean flow. The mean velocity was generated by a precursor RANS simulation in Rhea et al. (2009), while Uddin (2008) used a mean velocity profile given by Kays et al. (2004). Hallqvist (2006) studied the influence of inflow conditions on the accuracy of the simulations.

In the present study, the large eddy simulation method with two different subgrid-scale models is used to compute a turbulent upward water impinging jet flow with a nozzle to plate distance of $\mathrm{H}=10 \mathrm{e}$ and a Reynolds number of 16000 . The numerical simulation is compared with the previous experimental work done with the authors (Koched et al., 2011).

\section{Physical parameters}

\subsection{Flow configuration}

In the Cartesian coordinate system $(\mathrm{O} ; \mathrm{x}, \mathrm{y}, \mathrm{z})$, the computational domain is:

$$
\Omega=\left[-L_{x} / 2, L_{x} / 2\right] \times\left[0, L_{y}\right] \times\left[-L_{z} / 2, L_{z} / 2\right]
$$

where the origin $\mathrm{O}$ is located at the center of the plate and Lx, Ly and Lz magnitude are 85e, 10e and 40e, respectively (see Fig.1).

The Reynolds number of the flow is based on the jet width e and its average bulk velocity Um with

$$
R e=\frac{U_{m} e}{v}
$$

where $v$ is the (constant) kinematic viscosity. The nozzle-to-plate distance $\mathrm{H}$ corresponds to the computational domain height $\mathrm{Ly}$, with $\mathrm{H} / \mathrm{e}=10$.

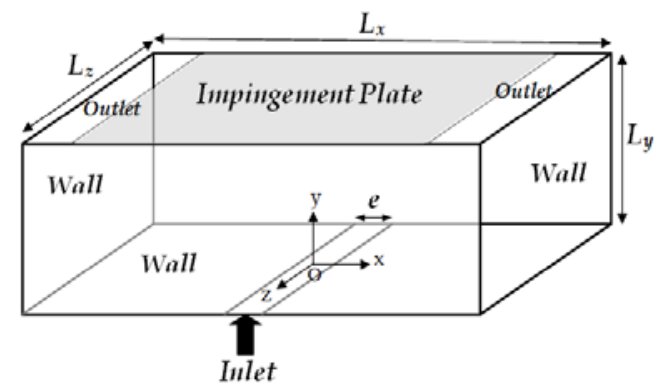

Fig. 1. Schematic representation of computational domain and boundary conditions.

This choice is due to compare the numerical results with the experimental work have done with the authors, previously. The jet and domain fluids are both water in standard condition. Referring to Koched et al. (2011), one can find more detail about the experimental setup and flow condition.

\subsection{Boundary conditions}

No-slip boundary conditions are applied to the impingement plate and to the other walls showed in Fig 1 . The turbulent velocity profile is used to inlet the jet flow to the domain. The constant pressure is used for the outlet boundary conditions.

\section{Numerical Scheme}

\subsection{Large eddy simulation method}

For incompressible flow, the space filtered continuity and Navier-Stokes equations (NS) can be written in the following non-dimensional form:

$$
\begin{aligned}
& \frac{\overline{\partial u_{i}}}{\partial x_{i}}=0 \\
& \overline{\frac{\partial u_{i}}{\partial t}}+\frac{\overline{\partial u_{i} u_{j}}}{\partial x_{j}}=-\frac{\overline{\partial P}}{\partial x_{i}}+\frac{1}{R e} \frac{\partial}{\partial x_{j}} \frac{\overline{\partial u_{i}}}{\partial x_{j}}-\frac{\partial \tau_{i j}}{\partial x_{j}}
\end{aligned}
$$

In present work, the equations are solved in three dimensions. All velocities and length scales are made non-dimensional by the inflow velocity and jet inlet diameter:

$$
\begin{aligned}
& L^{+}=\frac{u_{\tau} L}{v}, \\
& u^{+}=\frac{u}{u_{\tau}},
\end{aligned}
$$

$t^{+}=t \frac{u_{\tau}}{L}$,

where the friction velocity $u_{\tau}$ is calculated from the wall friction $\tau_{w}$ using the viscous shear stress:

$u_{\tau}=\sqrt{\frac{\tau_{w}}{\rho}}=\sqrt{v\left(\frac{\partial \bar{u}}{\partial y}\right)_{y=0}}$

Equations (3) and (4) govern the large resolved scale motion. The resolved scales interact with the unresolved scales via the SGS-stress:

$\tau_{i j}=\overline{u_{i} u_{j}}-\overline{u_{i}} \overline{u_{j}}$

\subsection{Sub-Grid Scale Models}

In the present work two different SGS models are used; a dynamic Smagorinsky model and a kinetic energy one-equation model.

The dynamic Smagorinsky model approximates the

SGS-stress as

$\tau_{i j}-\frac{1}{3} \delta_{i j} \tau_{k k}=-2 C \Delta^{2} \overline{|S|} \overline{S_{i j}}$

where

$\overline{S_{i j}}=\frac{1}{2}\left(\frac{\partial \overline{u_{i}}}{\partial x_{j}}+\frac{\partial \overline{u_{j}}}{\partial x_{i}}\right)$

$\overline{|S|}=\left(2 \overline{S_{i j}} \overline{S_{i j}}\right)^{1 / 2}$

In equation (10), $\mathrm{Sij}$ is the strain-rate tensor of the filtered velocity field, $\Delta$ is the filter width and $C$ is model parameter. It is well known that a clear improvement of the near-wall behavior of the Smagorinsky model can be obtained when a dynamic procedure is applied for the estimation of 
its "constant". (Dairay et al., 2014)

In dynamic model proposed by Germano et al. (1991) the constant C is not arbitrarily chosen (or optimized), but it is computed. This parameter is a function of space and time and is locally calculated in each time step and for each grid point using different levels of space filtering. Germano et al. (1991) introduced the smagorinsky as the following

$$
C=-\frac{L_{i j} M_{i j}}{2 M_{i j} M_{i j}}
$$

where Lij is the dynamic Leonard stresses and defined as

$$
L_{i j}-\frac{1}{3} \delta_{i j} L_{k k}=-2 C\left(\widehat{\Delta}^{2} \mid \widehat{\bar{S} \mid} \widehat{\overline{S_{i j}}}-\Delta^{2} \sqrt{\bar{S} \mid \overline{S_{i j}}}\right)
$$

and

$$
M_{i j}=\left(\widehat{\Delta}^{2}\left|\widehat{\bar{S} \mid} \widehat{\overline{S_{i j}}}-\Delta^{2}\right| \widehat{\bar{S} \mid \overline{S_{i j}}}\right)
$$

In above equations the grid filter and the second coarser filter (test filter) denoted by ${ }^{-}$and ${ }^{-}$, respectively.

As pointed out by Lilly (1992) Eq. (13) can either be used locally or it can be averaged over larger regions. If $\mathrm{C}$ is averaged over larger regions, certain local information is lost, but numerical stability is enhanced. On the other hand, if $\mathrm{C}$ is used locally, numerical instability may be caused by occasional large positive or negative values of $\mathrm{C}$. The instability can be avoided by enforcing local limits on C. An upper bound on C can be found from the total viscous stability condition on the explicit scheme employed here, taking into account molecular and SGS related viscosity. Here, the localized version of the dynamic Smagorinsky model proposed by Piomelli and Liu (1995) has been used.

The second SGS model is the turbulent kinetic energy one-equation model. The transport equation for SGS turbulent energy can be derived by first subtracting the filtered equations of motion from their exact non-filtered counterparts to give a relation for the fluctuating component of velocity $u^{\prime}$. Multiplying the result by the sub-grid velocity vector and contracting based on the assumption of SGS isotropy produces the one-equation turbulent energy model, one variant of which is given by Yoshizawa and Horiuti (1985).

$\frac{\partial}{\partial t}(k)+\frac{\partial}{\partial x_{i}}\left(U_{i} k\right)-\frac{\partial}{\partial x_{i}}\left(v_{e f f} \frac{\partial}{\partial x_{i}}(k)\right)=-B L-\frac{C_{e} k^{3 / 2}}{\Delta}$

where $\mathrm{k}$ is the turbulent kinetic energy and

$B=\frac{2}{3} k I-2 v_{s g s} \operatorname{div}(D)$

$v_{e f f}=v+v_{\text {turb }}$

The choice of kinetic energy one-equation model is motivated by several factors, chief among which is a comparative SGS model study done by Fureby et al. (1997). In an investigation of fully developed channel flows the one equation model is outperformed only by the full SGS stress model (which is computationally more expensive).

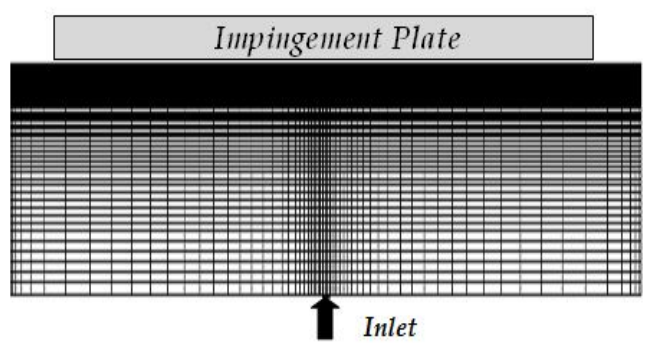

Fig. 2. 2D schematic representation of computational Grid.

\subsection{Discretisation of domain}

Meshes with hexahedral cells have been used in the computational domain. For these meshes, we have used the following non-dimensional cells sizes: $\left(\Delta x^{+}, \Delta z^{+}, \Delta y^{+}\right)=(10 \rightarrow 50,100,2 \rightarrow 10)$. In the wall normal directions, the non-dimensional cell sizes grow gradually from 2 at the wall to 10 in the bulk using a linear Stretching Ratio (SR) of 1.02. Like this, in transversal direction, the non-dimensional cell sizes grow from 10 to 50. Fig. 2 shows the schematic representation of impinging flow domain.

Table 1 Four case studies with different SGS models and different Courant number

\begin{tabular}{|c|c|c|}
\hline & SGS Model & $\begin{array}{c}\text { Courant } \\
\text { Number }\end{array}$ \\
\hline Case I & $\begin{array}{c}\text { Dynamic } \\
\text { Smagorinsky }\end{array}$ & 0.5 \\
\hline Case II & $\begin{array}{c}\text { Dynamic } \\
\text { Smagorinsky }\end{array}$ & 0.1 \\
\hline Case III & One-Equation & 0.5 \\
\hline Case IV & One-Equation & 0.1 \\
\hline
\end{tabular}

\subsection{OpenFOAM}

The OpenFOAM code (Weller et al., 1998) v.2.3.0 was used for the numerical simulations. The standard solver PisoFOAM was utilized for the incompressible LES modelling based on the finite-volume (FVM) factorized method (Geurts, 2004) and the predictor-corrector PISO (pressure implicit with splitting of operators) algorithm (Issa, 1986). Two and one iterations were set for a PISO loop and for non-orthogonal corrections, respectively. The generalized fully second order setup (in space and time) was used for all simulations.

The second order upwind scheme was applied for all convective terms approximation. All other inviscid terms and the pressure gradient were also approximated with a second order accuracy. A second order implicit Euler method (backward differentiation formula, BDF2 (Geurts, 2004) was used for the time integration together with the dynamic adjustable time stepping technique to 
guarantee the local Courant number less than $\mathrm{CFL}<$ 0.5 (for case I \& II) and $\mathrm{CFL}<0.1$ (for case III \& IV). Preconditioned (bi-) conjugate gradient method introduced by Hestens and Steifel (1952) with incomplete-Cholesky preconditioner (ICCG) by Jacobs (1980) was used for solving linear systems with a local accuracy of 10-7 for all dependent variables at each time step.

\section{Results and discussions}

\subsection{Mean velocity components}

Fig 3 and 4 show the LES simulation and the experimental results for transversal and axial mean velocity components, respectively. As shown in these figs, there is a good agreement between experimental data and the simulated results obtained from both SGS models.

Fig 3 reveals that the position of beginning of wall-jet region and also the boundary layer width along the impingement wall are predicted correctly using dynamic smagorinsky model in comparison with the experimental data (Koched, 2011). Also, the growth rate of turbulent jet is correctly predicted using SGS model (Fig. 4).
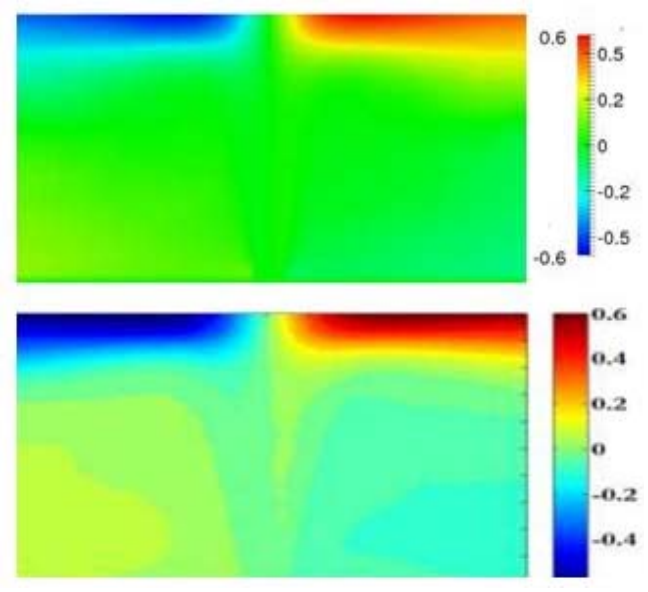

Fig. 3. transversal mean velocity component (m/s). Upper: Large eddy simulation with dynamic smagorinsky model, lower: experimental (Koched, 2011)
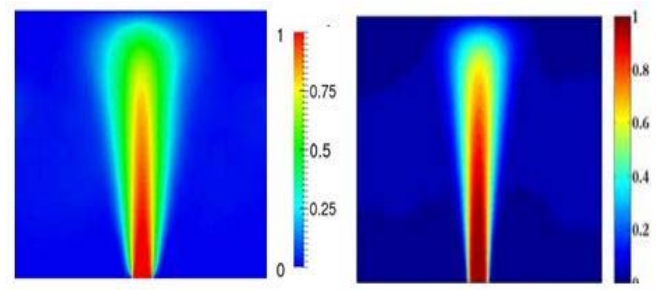

Fig. 4. Axial mean velocity component (m/s). Left: Large eddy simulation with dynamic smagorinsky model, Right: experimental (Koched, 2011)

\subsection{SGS Model Effects}

One way to evaluate the SGS-models is to perform LES with different SGS models and then compare the obtained turbulence statistics with DNS or LES with higher relaxation. This method is commonly referred to as a posteriori model testing. It is an expensive method for model evaluation due to the computational time required to achieve statistically stationary averages. The most important difference between a priori and a posteriori testing is that the a priori test is based on an instantaneous situation while a posteriori test is based on the time-averaged behavior. (Olsson and Fuchs, 1998)

Here, in order to evaluate the SGS-models, priori and posteriori tests are performed for both localized dynamic smagorinsky model and kinetic energy one-equation model. The one equation models account for non-equilibrium effects and provide an independent SGS velocity scale at a small increase in cost. This translates into increased accuracy, particularly in complex flow arrangements.

Fig 5 shows the instantaneous velocity contours for two SGS model at the same non-dimensional time. The effect of SGS model is obvious in both magnitude and direction of the velocity field. As shown in Fig 5, the velocity field is more diffuse in one-equation SGS model while the velocity magnitude in jet core is higher in localized dynamic smagorinsky model.

Fig 6 shows the comparison of the mentioned two SGS models with the experimental data providing by Koched et al. (2011) to present the capabilities of these models. Here, the transversal mean velocity component corresponding to the two different SGS models are compared with experimental data in three different horizontal planes; i.e., $\mathrm{y} / \mathrm{H}=0.08, \mathrm{y} / \mathrm{H}=0.5$ and $\mathrm{y} / \mathrm{H}=0.92$. As shown in Fig. 6, in near impingement wall region (at $\mathrm{y} / \mathrm{H}=0.92$ ), the localized dynamic smagorinsky model prediction for transversal component is closer to the experimental results compared with that of the one equation model. However, travelling toward the nozzle jet, the dynamic smagorinsky predictions improves. This may be attributed to the non equilibrium effects that are present in the impingement region.

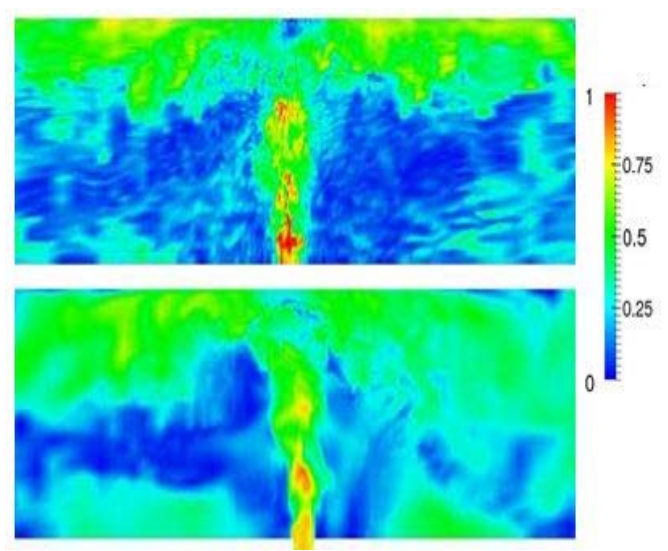

Fig. 5. Instantaneous velocity magnitude field using localized dynamic Smagorinsky (upper) and one-equation (lower) models 


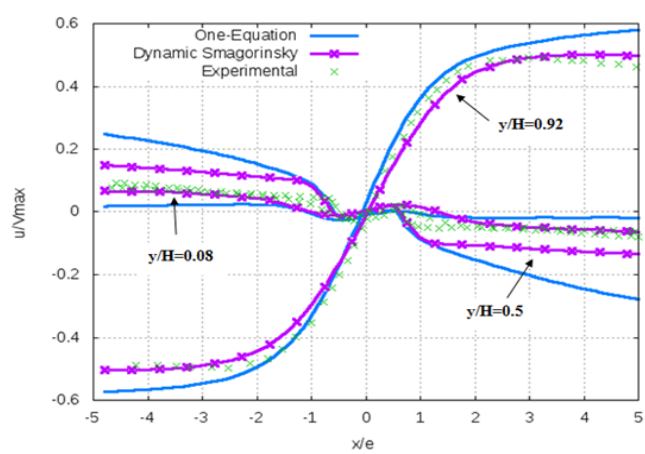

Fig. 6. Comparison of transverse profiles of the longitudinal and transversal mean velocity components between two SGS models and experimental data (Koched et al., 2011).

\subsection{Small scale effects}

In most of the simulations performed by numerous researchers using LES, the maximum CFL number is assumed to be 0.5 or 1 (Komen et al., 2014). However, the courant number can have significant effects on flow field characteristics. Due to large sensitivity of the simulation, the time steps selected turn out to impact the penetration curves.

Fig 7 shows the instantaneous velocity field resulting from two different maximum CFL numbers, namely, 0.5 and 0.1 . Both of these cases have similar computational grids but different time steps $(\Delta \mathrm{t})$. In case II $\left(\mathrm{CFL}_{\max }=0.1\right)$, the time step is $2 \times 10-6 \mathrm{~s}$ while the time step in case $\mathrm{I}\left(\mathrm{CFL}_{\max }=0.5\right)$ is about 10-5 s. As shown in Fig. 7, when the time steps reduce to about 10-6, the penetration of jet core velocity is increase. Fig 8 shows the effect of CFL number on the flow turbulent kinetic energy. The energy penetration in the flow domain for smaller CFL number is higher than that for bigger CFL numbers.

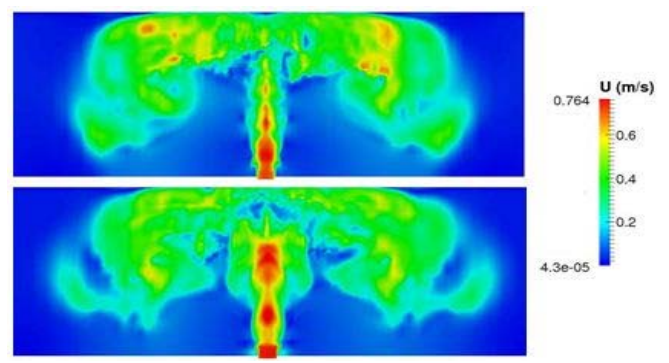

Fig. 7. Velocity magnitude contour for $\mathbf{C F L}=\mathbf{0 . 5}$ (upper) and $C F L=0.1$ (lower) in $(\operatorname{Re}=16,000)$.

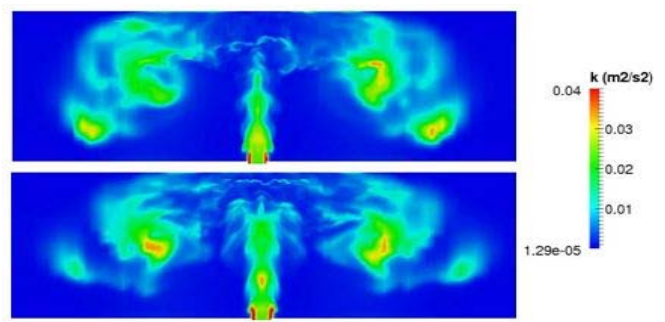

Fig. 8. Turbulent kinetic energy contour for CFL $=0.5$ (upper) and CFL=0.1 (lower) $(\operatorname{Re}=16,000)$.

\section{CONCLUSIONS}

A submerged turbulent water slot jet impinging normally on a flat plate has been numerically studied in three dimensions using two different subgrid scale models. The nozzle-to-plate distance was 10 nozzle jet width and the Reynolds number was 16000 . Comparative studies were done between the numerical simulation of impinging jet flow using large eddy simulation method and the relevant experimental data. The comparisons showed the high capabilities of the LES method to predict flow characteristic in both free-jet and impingement regions. In the second part of this paper, in order to investigate capabilities of different SGS models, priori and posteriori tests were performed. The relevant simulations were done with both the localized dynamic smagorinsky model and the turbulent kinetic energy one-equation model. The results revealed higher concurrence of the localized dynamic smagorinsky model results with the experimental data in simulating the subgrid structures in the impinging region, especially near the impingement wall and free jet region.

\section{REFERENCES}

Beaubert, F., and S. Viazzo. (2003) Large Eddy Simulations of Plane Turbulent Impinging Jets at Moderate Reynolds Numbers, International Journal of Heat and Fluid Flow 24, 512-519.

Cziesla, T., G. Biswas, H. Chattopadhyay, and N. K. Mitra (2001) Large-Eddy Simulation of Flow and Heat Transfer in an Impinging Slot Jet, International Journal of Heat and Fluid Flow $22,500-508$.

Dairay, T., V. Fortuné, E. Lamballais, and L. E. Brizzi. (2014) LES of a turbulent jet impinging on a heated wall using high-order numerical schemes, International Journal of Heat and Fluid Flow 50, 177-187.

Dewan, A., R. Dutta, and B. Srinivasan (2012) Recent trends in computation of turbulent jet impingement heat transfer, Heat Transfer Engineering 33, 447-460.

Fureby, C., G. Tabor, H. G. Weller, and A. D. Gosman. (1997), A comparative study of subgrid scale models in homogeneous isotropic turbulence, Physics of Fluids (1994-present) 9, 1416-1429.

Geurts, B., (2004). Elements of direct and large-eddy simulation, R. T. Edwards.

Germano, M., U. Piomelli, P. Moin, and W. H. Cabot, (1991). A dynamic subgrid-scale eddy viscosity model, Physics of Fluids A 3, $1760-1765$.

Gardon, R., and J. C. Akfirat, (1965). The Role of Turbulence in Determining the Heat Transfer Characteristics of Impinging Jets, International Journal of Heat Mass Transfer 8, 1261-1272.

Hallqvist, T., (2006). Large-eddy simulation of impinging jets with heat transfer, $P h D$ Thesis, 
Royal Institute of Technology, Department of Mechanics, Sweden.

Hadziabdic, M., and K. Hanjalic, (2008). Vortical Structures and Heat Transfer in a Round Impinging Jet, Journal of Fluid Mechanics 596, 221-260.

Hestens, M., and E. Steifel, (1952). Methods of conjugate gradients for solving systems of algebraic equations, $J$ Res Nat Bur Stand 29, 409-36.

Issa, R., (1986). Solution of the implicitly discretized fluid flow equations by operator splitting, Journal of Computational Physiscs, 62 , 40-65.

Jacobs, D., (1980) Preconditioned conjugate gradient methods for solving systems of algebraic equations, Technical report, Central Electricity Research Laboratories, Leatherhead, Surrey, England.

Jambunathan, K., E. Lai., M. A. Moss., and B. L. Button (1992) A Review of Heat Transfer Data for Single Circular Jet Impingement, International Journal of Heat Fluid Flow, 13, 106-115.

Kays, W. M., M. E. Crawford., and B. Weigand (2004), Convective Heat and Mass Trans., McGraw-Hill, New York.

Koched, A., M. Pavageau., and F Aloui. (2011) Vortex structure in the wall region of an impinging plane jet, Journal of Applied Fluid Mechanics, 4, 61-69.

Koched, A. (2011) Analyse expérimentale multi-échelles des interactions entre un écoulement et une paroi par PIV et polarographie : Application à un jet plan immergé et confiné en impact. PhD Thesis, École des Mines de Nantes, France.

Lilly D. K. (1992) A proposed modification of the germano subgrid-scale closure method, Physiscs, Fluids A, 4, 633.

Lodato G., L. Vervisch., and P Domingo. (2009) A Compressible Wall-Adapting Similarity Mixed
Model for Large-Eddy Simulation of Impinging Round Jet, Physics of Fluids, 21, 1-21.

Martin, H. (1977) Heat and Mass Transfer Between Impinging Gas Jets and Solid Surfaces, Advanced Heat Transfer, 13, 1-60.

Olsson, M. and L. Fuchs. (1998) Large Eddy Simulations of a Forced Semi confined Circular Impinging Jet, Physics of Fluids, 10, 476-486.

Piomelli, U. and J. Liu.(1995) Large-eddy simulation of rotating channel flows using a localized dynamic model, Physics of Fluids A, 7, 839-848.

Piomelli, U. and J. Chasnov (1996) Large Eddy Simulations: Theory and Applications in Turbulence and Transition Modelling, Kluwer Academic Publisher, Dordrecht, the Netherlands, 269-336.

Hadziabdic, M. and K. Hanjalic. (2008). Vortical Structures and Heat Transfer in a Round Impinging Jet, Journal of Fluid Mechanics, 596, 221-260.

Rhea, S., M Bini., M Fairweather., and W. P Jones. (2009) RANS Modeling and LES of a Single-Phase Impinging Plane Jet, Computers \& Chemical Engineering, 33(8), 1344-1353.

Uddin, N. (2008) Turbulence Modeling of Complex Flows in CFD, PhD. Thesis, Universtat Stuttgart, Institute of Aerospace Thermodynamics, Stuttgart, Germany.

Voke P. R., and S. Gao. (1998) Numerical Study of Heat Transfer From an Impinging Jet, International Journal of Heat and Mass Transfer, 41, 671-680.

Yoshizawa A., and K. Horiuti. (1985). A statistically-derived subgrid-scale kinetic energy model for the large-eddy simulation of turbulent flows, Journal of the Physical Society of Japan, 54, 2834-2839.

Zuckerman N., and N. Lior., (2006) Jet impingement heat transfer: Physics, correlations, and numerical modeling, Advances in heat transfer, 39, 565-631. 